\title{
A Pre-Scanning Fast Handoff Scheme for VoIP in WLANs
}

\author{
Jun Xiao and Feng Liu \\ School of Computer and Information Technology, Beijing Jiao tong University \\ Beijing 100044, China \\ \{09112070,fliu\}@bjtu.edu.cn
}

\begin{abstract}
IEEE 802.11 wireless local area networks (WLAN) have been widely deployed, and many applications are implemented on WLAN. As one of the interesting wireless services, wireless voice over internet protocol (VoIP) can provide low-cost real-time voice service to mobile users. But the IEEE 802.11 wireless network cannot satisfy the QoS of VoIP. The delay during handoff process can last up to 1 second. Applications with delay restraint like VoIP will drop the call when the delay is more than $50 \mathrm{~ms}$. Therefore this paper analyzed the delay made by the handoff process, and proposed a pre-scanning fast handoff scheme. This fast handoff scheme is based on selective scanning and caching. It can predict the next AP for STAs and use pre-scanning before the handoff is triggered. Simulations on OPNET show this proposed scheme can reduce the average handoff delay to $23.45 \mathrm{~ms}$ and avoid some unnecessary handoffs. The pre-scanning fast handoff scheme is feasible and economical according to its performance in the handoff process.
\end{abstract}

Keywords: handoff, scanning, VoIP, QoS, IEEE 802.11

\section{Introduction}

IEEE 802.11 wireless local area networks (WLAN) have been widely deployed in recent years. It can provide wireless internet connection in many places such as home, office rooms, and commercial centers, not only in buildings but also in parks, streets and vehicles. Many mobile devices such as laptops, tablet computers, and cell phones have integrated with IEEE 802.11 equipment. As wireless networks becoming more accessible, many interesting wireless applications and services are provided to users. Voice over internet protocol (VoIP) over wireless networks, which is also called Voice over Wireless LAN (VoWLAN), is one of the most interesting wireless services. It can provide assorted and low-cost real-time voice services for mobile users. Through VoIP is highly-developed over wired networks in past, VoWLAN has some new problems which are pressing for solution.

IEEE 802.11 networks consist of four major physical components. The components are stations (STA), access points (AP), wireless medium and distribution system. Stations are computing devices with wireless network interfaces. Access points can perform the wireless-to-wired bridging function. Wireless medium is used to move frames from station to station. Distribution system is the logical component used to forward frames to their destination. The basic building unit of an 802.11 network is the basic service set (BSS), which is simply a group of stations that communicate with each other. Chaining BSSs together with distribution system creates extended service set (ESS). IEEE 802.11 standard [1] has two operating modes, infrastructure and ad-hoc mode. The infrastructure mode distinguished by the use of an access point is the most common form of deployments. In infrastructure networks, APs are used in all communications, including the communications between mobile stations in the same BSS. If one STA in infrastructure BSS wants to connect with another STA, the communication must take two hops. Firstly, this STA transfers the frame to the AP. Then the AP transfers the frame to 
the destination STA. This mode can reduce configuration and energy. But it limits the use area of WLAN, since APs have a small range. Now the optimization of IEEE 802.11 networks deployments for performance still remains work in progress.

Once a STA moves out of the range of current AP in a BSS, it needs a new AP and associate with it. This process is called layer-2 handoff or MAC layer handoff. A typical handoff scenario descripted above is shown in Figure 1. When a STA is moving away from the current AP, the signal strength and the signal-to-noise ratio (SNR) of the signal from the AP decrease. Sometimes, a handoff is also triggered when other metrics like the retry rate of data packets cannot be used. Then the STA breaks its association with the current AP, and scans a new AP to associate with. Essentially, during the handoff process, the STA's communication is suspended and all incoming packets will be dropped. Only management frames are exchanged between the STA, the current AP and the adjacent APs before the STA is able to associate with a newly selected AP. Depending on different handoff strategy, the delay during handoff process can last up to 1 second or more. Applications with delay restraint like VoIP will drop the call when the delay is more than $50 \mathrm{~ms}$ [2]. So the handoff strategy must be improved for the real-time applications.

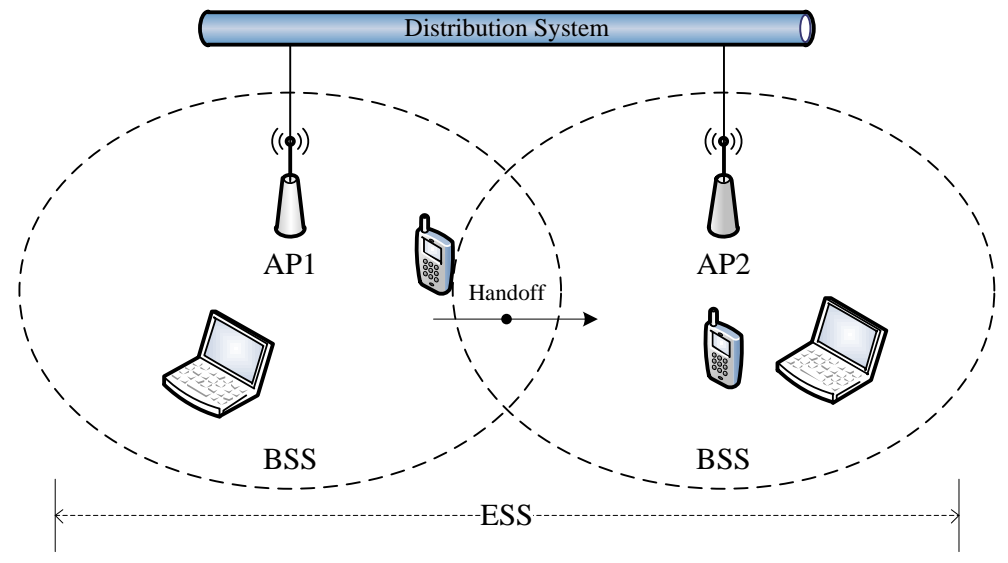

Figure 1. A Typical Handoff Scenario

So this paper analyzed the handoff process, and found out the time-consuming phase in the handoff process. Then a pre-scanning fast handoff scheme is proposed. It can reduce the average delay in MAC layer handoff to no more than $30 \mathrm{~ms}$, which agrees with the real-time applications like VoIP. This fast handoff scheme can be deployed in existing mobile devices, while the existing APs do not need any adaptation. In the simulations, the proposed handoff scheme not only can reduce delay in handoff process, but also the number of handoffs is decreased, which means some unnecessary handoffs are avoided. So this scheme is feasible and economical for VoIP in WLANs.

The rest of this paper is organized as follows. Section 2 presents the IEEE 802.11 background and the related work, while the analysis of handoff process is presented in this section. Section 3 provides an operation detail of the pre-scanning fast handoff scheme. In Section 4, some simulations are implemented and the results are discussed. Section 5 concludes this paper.

\section{Background and Related Work}

The basic handoff process consists of three phases: scanning, authentication and reassociation. Scanning can be accomplished either in passive or active mode according to the IEEE 802.11 standard. This phase is used for STA to find out a best next AP. When 
the best next AP is selected, the STA will do the next two phases and finish this handoff process.

Authentication is a phase in which the AP will decide whether to accept the identity of the STA. In this process the STA sends an authentication request frame carrying the identity information to the AP. Then the AP responds with an authentication response frame, indicating acceptance or rejection for the STA.

Re-association phase is after the successful authentication, the STA sends a reassociation request to the new AP, which will then send a re-association response containing acceptance or rejection to the STA.

In these three phases, the scanning delay is the dominating component which accounts for more than $90 \%$ of the overall handoff delay [3].

In passive mode, the STA listens to the wireless medium for beacon frames in each channel, which provide a combination of timing and control information to STAs. Using the information and the signal strength of beacon frames, the STA selects an AP to join. But STAs need to stay on each channel at least for one beacon interval to listen to beacons from all APs on this channel. So the passive mode passive scanning delay $D p s$ is given by:

$$
D_{p s}=N \times T_{B I}+N \times \delta
$$

Where $\delta$ is the time taken to switch the radio from one channel to another, $N$ is the number of channels in the IEEE 802.11 spectrum, and TBI is the beacon interval which is usually about $100 \mathrm{~ms} ; \delta$ is varying from 5 to $10 \mathrm{~ms}$ depending on the vendor implementation. So passive scanning yields a delay of more than 1 second to scan all 11 channels in IEEE 802.11b networks. Obviously it is not suited for delay-sensitive voice traffic. Hence active scanning is more useful.

In active scanning mode, the STA to broadcast probe requests evoking probe responses from APs actively without waiting for any beacon frames. The active scanning process is as following steps which are depicted in Fig. 2. Firstly The STA in the scanning phase broadcasts a probe request frame to APs in a channel and sets on a probe timer. Then listening for probe responses, if no response has been received by minChannelTime, the STA will probe the next channel. If one or more responses are received by minChannelTime, the STA will continue accepting probe responses until maxChannelTime. Next, the STA moves to the next channel and repeats the above steps. After all channels have been scanned, all information received from probe responses is processed so that the STA can select a best AP to join next. So the active scanning delay $D A S$ is given by:

$$
N \times \text { minChannel Time }+N \times \delta \leqslant D_{A S} \leqslant N \times \text { maxChannel Time }+N \times \delta
$$

Where $\delta$ is the time taken to switch the radio from one channel to another, $N$ is the number of channels in the IEEE 802.11 spectrum, minChannelTime and maxChannelTime are set by STA for waiting for response frame. $\delta$ is varying from 5 to $10 \mathrm{~ms}$ depending on the vendor implementation. The minChannelTime and maxChannelTime are tunable parameters and various studies ([3] and [4]) have suggested them. If minChannelTime is $5 \mathrm{~ms}$ and maxChannelTime is $11 \mathrm{~ms}$, the active scanning varies from $110 \mathrm{~ms}$ to $176 \mathrm{~ms}$ in IEEE $802.11 \mathrm{~b}$ networks. So active scanning is much faster than passive scanning and more widely used in practice for handoff situations. But it does not satisfy the delay time constrain of VoIP. 


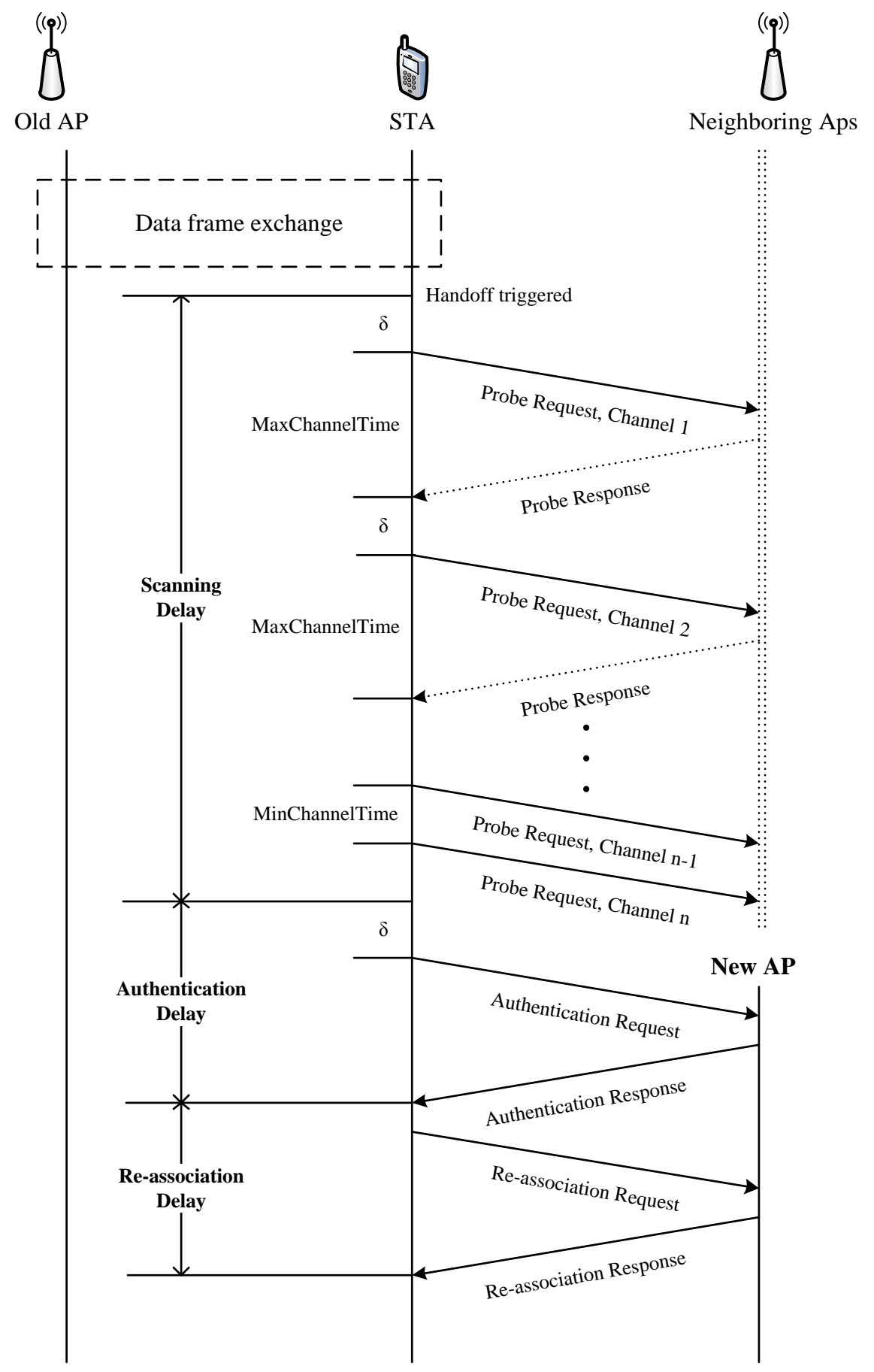

Figure 2. IEEE 802.11 MAC Layer Handoff Process with Active Scanning

Once scanning is over and the best AP has been identified, the STA will start the authentication phase and re-association phase. The authentication phase will cause about $6 \mathrm{~ms}$ delay which is different between various vendors. And the re-association phase will cause about 4 ms delay. IEEE 802.11r [5] is implementing extensions to the IEEE 802.11 base specification to directly support fast handover in the MAC protocol. With IEEE 802.11r, the initial AP acts as the authenticator, communicating with the AAA server. After that, each AP interacts with the initial AP rather than directly with the AAA server [6]. So IEEE $802.11 \mathrm{r}$ can reduce the authentication delay and re-association delay. Therefore the aggregate handoff delay is given by: 


$$
D_{\text {handoff }}=D_{\text {Scaning }}+D_{\text {Authentica tion }}+D_{\text {Re-associa tion }} .
$$

The average handoff delay is about $200 \mathrm{~ms}$ in an IEEE $802.11 \mathrm{~b}$ network with 11 channels and active scanning. It cannot satisfy the real-time applications like VoIP whose delay requirement is no more than $50 \mathrm{~ms}$. The scanning delay is the main component in handoff delay. So many researches on fast (or seamless) handoff in IEEE 802.11 are focused on reducing the scanning time and want to find out the potential optimizations of the scanning delay.

Calculating suitable values of minChannelTime and maxChannelTime for handoffs is proposed earlier. This approach did not alter the original IEEE 802.11 handoff strategy. MinChannelTime represents the minimum duration a STA must wait after sending out a probe request and before deciding that the channel is empty. If MinChannelTime is too small, the STA will not find any AP in this channel. While if it is too long, the STA will waste time in empty channel. In [3] and [4], the suitable MinChannelTime value is from $1 \mathrm{~ms}$ to $7 \mathrm{~ms}$. MaxChannelTime is the maximum duration a STA has to wait for probe responses from APs in this channel. This value should ensure that probe responses from most or all of the APs are collected by the STA before moving to the next channel. This value is depends on the AP density in each channel. This value should be naturally minimized to reduce the scanning phase, but it may result in candidate APs being missed. In [3] and [4], the suitable MaxChannelTime is around $11 \mathrm{~ms}$ which can suffice in practice to capture most or all probe responses. This approach can reduce the delay, but in AP dense networks the delay is also too high to support the real-time applications.

In [7] and [8], they proposed a selective scanning approach which scanning some channels rather than all channels. APs operate on orthogonal channels to minimize adjacent channel interference (e.g. only 3 such channels for IEEE 802.11b). Each BSS is typically surrounded by a few APs operating on a subset of the total number of channels. Clearly, there is no need to scan all $802.11 \mathrm{~b}$ channels, as some channels would just be devoid of APs. Based on previous scans, STAs store the information about the best AP for each neighboring channel. This approach scans fewer channels than the traditional approach, so it reduces much more time on scanning. But it depends too much on the WLAN topology. If the topology is changed, this approach will be invalid.

Using multiple radios is proposed in [9]. In this approach a STA needs at least two radios, one of which is reserved for scanning, and the others are used for normal operation. The STA can exchange data frames with a serving AP while it performs scanning. So this approach can realize a seamless handoff. However, since typical commercial STAs, such as cell phone, are not equipped with multiple radios. Anymore, only for scanning, using multiple radios will waste more energy.

Another approach is using GPS for movement detection, which can exactly compute STAs' site and pick out the suitable next APs for them. In [10], an efficient GPS-based handoff scheme is designed for vehicular networks. AP selection scheme can find the best AP within a vehicle's transmission range for a handoff, which is located on the vehicles potential path so that it can maintain a long and stable connection with the vehicle. This approach significantly reduces the time and the number of handoffs using GPS in vehicular networks. However GPS is not suitable for indoors networks.

A pre-scanning fast handoff scheme will be proposed in this paper to reduce the handoff delay. This scheme will be discussed below. 


\section{Pre-scanning fast handoff scheme}

Since the analysis has been presented in Section 2, the scanning phase caused the dominating delay in handoff. So the proposed pre-scanning fast handoff scheme is mainly work on the improvement of scanning phase. This scheme is based on selective scanning and caching. It consists of scanning trigger, channel scanner, handoff trigger, and caching. The process of the pre-scanning fast handoff scheme is showed in Figure 3. The solid line is controlling flow, and the dart line is data flow.

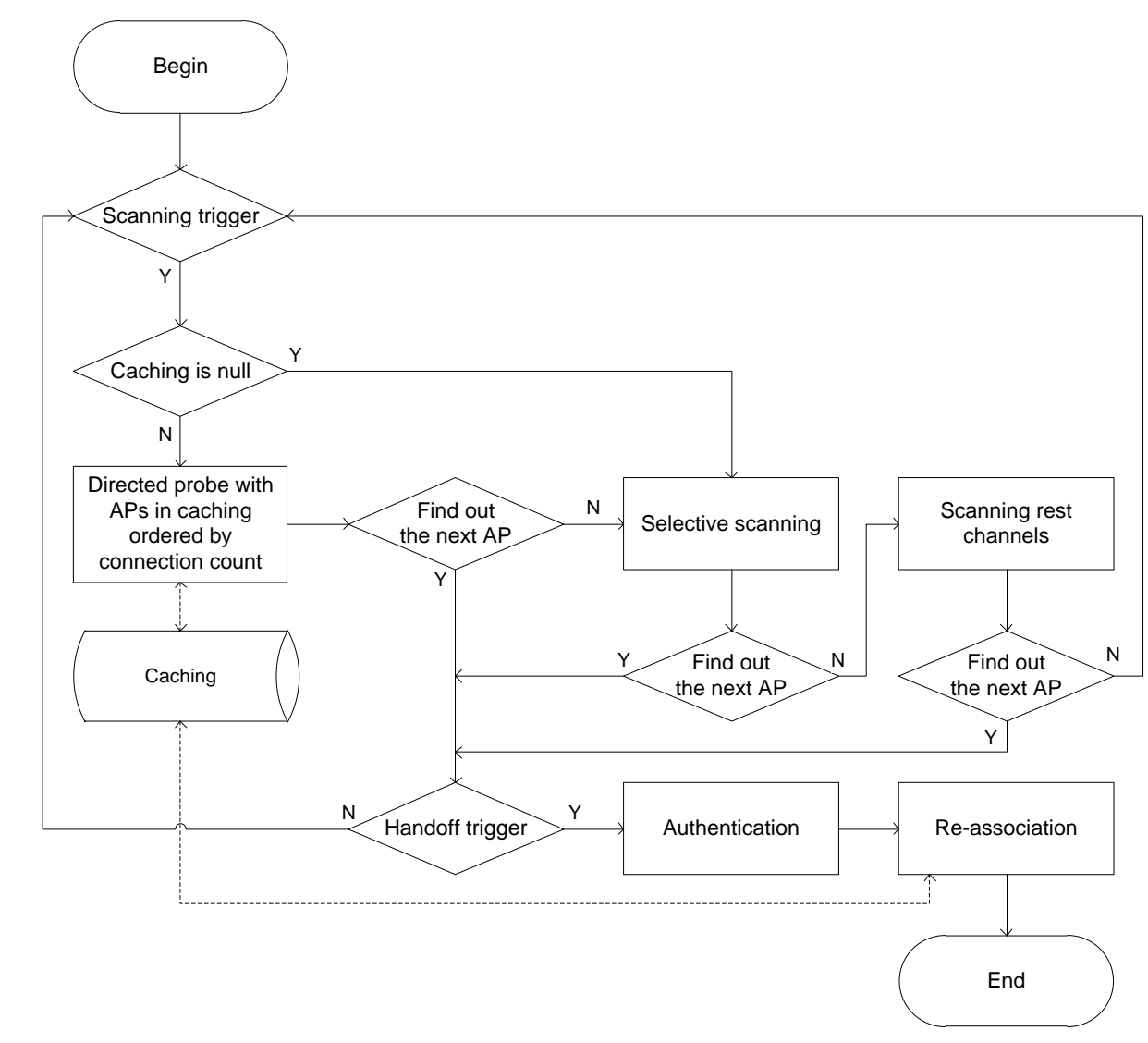

Figure 3. The Process of the Pre-scanning Fast Handoff Scheme

\subsection{Scanning Trigger}

The scanning trigger is different from the handoff trigger. Scanning trigger is more sensitive than handoff trigger. When the STA does not transmit real-time package or make any call with others in the VoIP application, scanning will be implemented before the handoff is triggered. This is called as pre-scanning. In this paper, RSSI and an accelerometer are used as scanning trigger. A STA scans channels only when the RSSI from its current AP is below a specified threshold and it is moving.

RSSI is a reasonable handoff trigger. STAs implement handoff mainly for they are moving out of the range of current AP. The distance between the STA and its current AP directly represents the need of handoff for the moving STA. RSSI is inversely proportional to the distance. Assuming a STA is moving from AP1 to AP2, when the STA is moving far away from AP1, the RSSI of AP1 is decreasing gradually. Conversely, when the STA is closing with AP2, the RSSI of AP2 is increasing gradually. So RSSI is a suitable trigger reflected the movement of STAs.

The accelerometer is a motion sensor which is already deployed in many commercial smartphones. It is accurate and energy efficient [11]. A STA needs a handoff when it is 
moving from a current AP to another AP. So an accelerometer can be used to detect the motion status of a STA, only when it is moving and the RSSI is below the threshold, the STA will implement a handoff.

\subsection{Channel Scanner}

The STAs learn about available APs by scanning other IEEE 802.11 channels for available APs in the same WLAN. Scanning other IEEE 802.11 channels can be performed actively or passively. This scheme used the active scanning.

In active scanning, a STA switches to a channel, sends a probe request, and then waits to hear any probe responses from APs on that channel. The probe-request frames used in an active scan are two types. First one is directed probe, in which the STA sends a probe request with a specific destination SSID, and only APs with a matching SSID will reply with a probe response. Another is broadcast probe, in which the STA sends a broadcast probe request, and all APs receiving the probe-request will respond with a probe-response for each SSID they support.

In pre-scanning fast handoff scheme, directed probe is used when there are candidates of the next AP from the current AP in the STA's caching. The STA will probe those APs directly in the order from high connection count to low count. The probe method can use the handoff history information of a STA. And it will not make networks congested. After a handoff, the connection count of the AP, which next associates with the STA, will plus one in caching.

When the directed probes are all failed or the caching is null, selective scanning, which is often used to reduce the handoff delay by many other methods, will be used. In IEEE 802.11b networks, only three channels (channel 1, 6 and 11) are not overlapping in Fig. 4. Therefore, most of APs in a WLAN are configured with these non-overlapping channels to avoid channel interference. Scanning these channels firstly can have more chance of finding out the next AP and save more time than full scanning, since a STA only scan three channels rather than 11. When a next AP is not found in these channels, the STA will scan the rest channels.

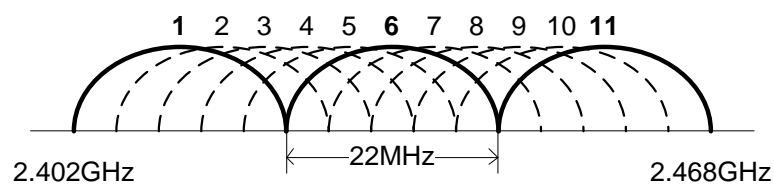

Figure 4. Channel Frequency Distribution in IEEE

\subsection{Handoff Trigger}

The handoff trigger in IEEE 802.11 standard is used for a STA to decide making a handoff in current connection. Handoff trigger is an important component in handoff algorithm. If the handoff trigger is too sluggish, STAs will suffer an unaccepted latency when they cannot connect to new APs immediately. While if the handoff trigger is too sensitive, the STA will make unnecessary handoff so frequently that it will cause congestion in a busy network and consume more power.

In the pre-scanning fast handoff scheme, the handoff threshold is lower than scanning threshold. If the scanning is finished and the best next AP is found out, the STA will implement handoff directly without scanning. Otherwise the STA will wait for scanning and then implement handoff.

\subsection{Caching}

Caching is used for recording the APs which are scanned and associated by the STA, and offers useful information for channel scanning. 
When a scanning is finished, the scanned APs will be put into caching table shown in Table. 1. The current AP is the AP that the STA is current connecting to. And the scanned AP is the AP scanned by the STA when the scanning threshold is triggered. For example, when the RSSI of the moving STA from AP1 is below the scanning threshold, the STA scans and finds AP2, AP3 and AP4. So there are three entries recorded in caching table. The AP information in every entry includes MAC address, SSID and other useful information about this AP.

When a handoff is completed, the connection count of the new associated AP in caching table will increase one. For example, when a STA finished a handoff from AP1 to AP2, the connection count in the entry about AP1 and AP2 will be added one.

When a new channel scanning is triggered, the STA will search caching table, find out entries about the current AP, order connection count from high count to low count, and scan these AP orderly by directed probe. For example, when a STA reaches the scanning threshold with the current AP1, it will find out three entries about AP1 in caching table. And it will scan as this sequence, $\mathrm{AP} 2 \rightarrow \mathrm{AP} 3 \rightarrow \mathrm{AP} 4$, until a suitable AP replies with probe response frame.

Table 1. Caching Table Structure

\begin{tabular}{|c|c|c|}
\hline Current $\boldsymbol{A P}$ & Scanned $\boldsymbol{A P}$ & Connection Count \\
\hline AP1 & AP2 & 3 \\
\hline AP1 & AP3 & 1 \\
\hline AP1 & AP4 & 0 \\
\hline$\ldots$ & $\ldots$ & $\ldots$ \\
\hline APn & APm & k \\
\hline
\end{tabular}

\section{Simulation and Results Analysis}

This section presents the performance evaluation of the pre-scanning fast handoff scheme, and compares its accuracy and average delay against using full scanning and non-overlapping scanning in IEEE 802.11b network.

\subsection{Simulation Environment}

Simulations are implemented on OPNET Modeler 14.5 which is a commercial network simulation tool.

An IEEE 802.11b wireless network is deployed like Figure 6. There are ten APs which are deployed with non-overlapping channels and 10 STAs which can move among the 36 blocks. The trajectories of the STA are created by a computer procedure. There are three trajectory types, cycle, return and random shown in Figure 6. In each trajectory type, every STA will go through 50000 edges of these blocks about 2500 hours. A prescanning fast handoff process model is created on OPNET for realizing the proposed prescanning fast handoff scheme. To make comparisons, full scanning and non-overlapping scanning process models are also created. The delay parameters used in simulations are shown in Table 2. 




Figure 6. The Deployment of IEEE 802.11b Network and Three Trajectory Types

Table 2. Delay Parameters Used in Simulations

\begin{tabular}{|c|c|}
\hline Parameters & Delay \\
\hline MaxChannelTime & $200 \mathrm{~ms}$ \\
\hline MinChannelTime & $20 \mathrm{~ms}$ \\
\hline Channel switching time & $11.4 \mathrm{~ms}$ \\
\hline Authentication delay & $6 \mathrm{~ms}$ \\
\hline Re-association delay & $4 \mathrm{~ms}$ \\
\hline
\end{tabular}

\subsection{Simulation Results}

The simulation results are shown in Figure 7. In Figure 7 (a), the average delay of handoffs is compared between the pre-scanning, full scanning and non-overlapping scanning. The average handoff delay of pre-scanning fast handoff scheme is about 23.65 $\mathrm{ms}$. In cycle trajectory, the average delay is $23.71 \mathrm{~ms}$. In return trajectory, the average delay is $24.58 \mathrm{~ms}$. and in random trajectory, the average delay is $22.69 \mathrm{~ms}$. It can reduce the handoff delay vastly comparing to the other two methods with which the average delays are about $885.40 \mathrm{~ms}$ and $634.20 \mathrm{~ms}$ respectively. So The QoS about delay in realtime applications like VoIP can be satisfied with the proposed pre-scanning fast handoff scheme. While in Figure 7 (b), the number of handoffs is also decreased by nearly $2 \%$ with the pre-scanning fast handoff scheme. Avoiding unnecessary handoffs, the STA can save power energy. According to the simulations, the proposed fast handoff scheme is useful and economical. 


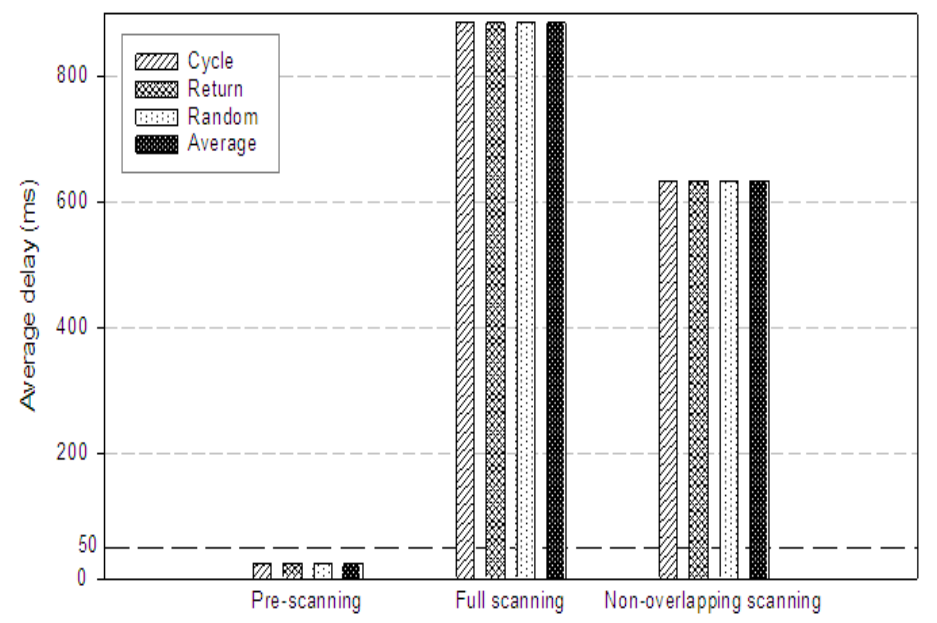

(a)

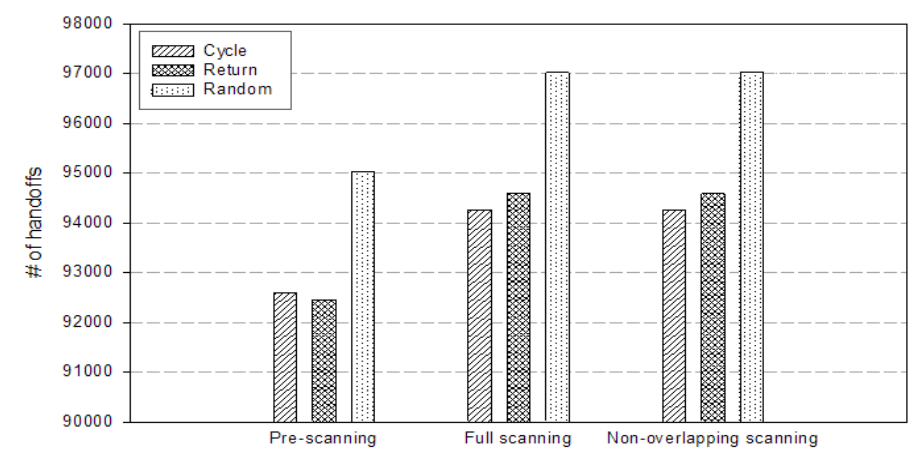

Figure 7. Simulation Results: (a) Average Delay; (b) The Number of

In the simulations, the next AP prediction accuracy with the pre-scanning fast handoff scheme is shown in Figure 8 (a). The overall accuracy with the pre-scanning fast handoff scheme is $99.68 \%$. The overall accuracy for cycle trajectory is $99.68 \%$. The overall accuracy for return trajectory is $99.69 \%$. And the overall accuracy for random trajectory is $99.67 \%$. The failed proportion is about $0.32 \%$ because when a STA moves into a new network which it has not scanned there is no entry about the APs of the new network in the caching of the STA. Therefore the predictions are failed without the supporting information which has not been initialized in the caching about new networks. Except the initialization, the prediction accuracy is $100 \%$, which means the next AP prediction is guaranteed to be correct. However, the accuracy of each prediction is important because a mis-prediction increases the handoff delay by $\delta+$ tresponse, which is more than $10 \mathrm{~ms}$. So when the 4th prediction is failed, adding the delay of authentication and reassociation, the handoff delay will more than $50 \mathrm{~ms}$. The average $1 \mathrm{st}$ prediction accuracy is $44.38 \%$. When 1 st prediction is failed, the 2 nd prediction, whose average accuracy is $29.22 \%$, will do its work. The average 3rd prediction accuracy is $16.68 \%$. The combined average accuracy of the first three predictions is about $90.28 \%$. While the combined average accuracy of the beyond 4th predictions is nearly $9.40 \%$. Adding the failed proportion, $9.32 \%$ handoffs will product more than $50 \mathrm{~ms}$ delay. In Figure 8 (b), the proportion of handoff delay which is more than 50 $\mathrm{ms}$ is $4.77 \%$ in whole handoffs, not $9.40 \%$, because of using pre-scanning. There is $50 \%$ chance to using pre-scanning in these simulations, which means the real-time application is mute in half handoffs. 
To reduce the delay of the $0.32 \%$ handoffs with failed predictions, the small values of minChannelTime and maxChannelTime can be used, because if the predictions are failed the whole handoff process is as same as the non-overlapping scanning handoff. However, to reduce the delay of the $90.28 \%$ handoff with beyond 4 th predictions, it is critical to increase the 1 st prediction accuracy. The next work is to find a method to increase the prediction accuracy for reducing the handoff delay.

\section{Conclusion}

This paper analyzed handoff process and found out that the scanning phase produced the dominating delay. So this paper proposed a pre-scanning fast handoff scheme to reduce the handoff delay. This pre-scanning fast handoff scheme is based on the selective scanning and caching. It can predict the next AP for STAs and use pre-scanning before the handoff is triggered. Its process model had been created on OPNET platform, and to make comparisons, the full scanning and non-overlapping scanning process model were all created.


Figure 8. The Next AP Prediction Accuracy and Delay Proportion:

(a) The Next AP prediction Accuracy; (b) The Delay Proportion in Whole Handoffs

The sufficient simulations were implemented on OPNET platform. From the simulation results, the proposed handoff scheme can reduce the average handoff delay to around $23.45 \mathrm{~ms}$ and avoid some unnecessary handoffs. It is feasible and economical according to its performance on handoffs. The future work, two issues are planned to investigate. First, for increasing the prediction accuracy, the handoff history information must be used efficiently in prediction process. Second issue is finding out a fast handoff scheme to speed up vertical handoffs in heterogeneous networks.

\section{Acknowledgements}

The authors would like to thank the reviewers for their detailed reviews and constructive comments, which have helped improve the quality of this paper. This work was supported by National Key Scientific Instrument and Equipment Development Projects of China under Grant No. 2013YQ33066705.

\section{References}

[1] "IEEE Standard 802.11Part 11: Wireless LAN medium access control (MAC) and physical layer (PHY) specifications”, ANSI/IEEE STD 802.11, 1999 edition (R2003), (2003), pp.1-513.

[2] "General characteristics of international telephone connections and international telephone circuits", International Telecommunication Union, ITU-TG. (1988) p.114. 
[3] A. Mishra, M. Shin, and W. Arbaugh, "An Empirical Analysis of the IEEE 802.11 MAC Layer Handoff Process", ACM SIGCOMM Computer Communication Review, vol. 33, no. 2, (2003), pp.93-102.

[4] H. Velayos and G. Karlsson, "Techniques to Reduce the IEEE 802.11b Handoff Time", IEEE International Conference on Communication (ICC ‘04), (2004) June, Paris, France.

[5] IEEE 802.11r. "Wireless LAN Medium Access Control and Physical Layer Specification: Amendment for Fast BSS Transition", (2008).

[6] T. C. Clancy, "Secure Handover in Enterprise WLANs: Capwap, Hokey, and IEEE 802.11R", Wireless Communications, IEEE, vol. 15, no. 5, (2008) October, pp.80-85.

[7] H. Purushothaman and R. Sumit, "Fast Scan: a Handoff Scheme for Voice over IEEE 802.11 WLANs", Wireless Networks, vol. 16, no. 7, (2010) October, pp. 2049-2063.

[8] S. Shin, A. G. Forte, A. S. Rawat, and H. Schulzrinne," Reducing MAC layer handoff latency in IEEE 802.11 wireless LANs", The second international workshop on Mobility management and wireless access protocols (MobiWac '04), ACM, Philadelphia, PA, USA, (2004) September 26 - October 1, pp.19-26.

[9] S. Jin and S. Choi, "A Seamless Handoff with Multiple Radios in IEEE 802.11 WLANs", IEEE Transactions on Vehicular Technology, vol. 63, no. 3, (2014), pp.1408-1418.

[10] M. Almulla, Y. Wang, A. Boukerche, and Z. Zhang," A Fast Location-based Handoff Scheme for Vehicular networks", IEEE International Conference on Communications (ICC '13), Budapest, Hungary, (2013) June, pp.1464-1468.

[11] I. Shafer and L. M. Chang, "Movement Detection for Power-Efficient Smartphone WLAN Localization", Proc. of the 13th ACM international conference on Modeling, analysis, and simulation of wireless and mobile systems (MSWIM '10), Bodrum, Turkey, (2010) October 17-2, pp.81-90.

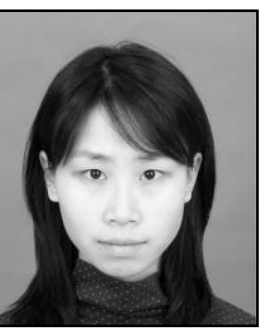

\section{Authors}

Jun Xiao, is currently a Ph.D candidate at Beijing Jiaotong University, Beijing, China. She received her B.S. and M.S. degree in Computer Science and Technology from Beijing Jiaotong University, Beijing, China, respectively in 2007 and 2009. Her research interests include quality of service in wireless networks, VoIP, VoWLAN, and high speed railway information technology. Email: 09112070@bjtu.edu.cn

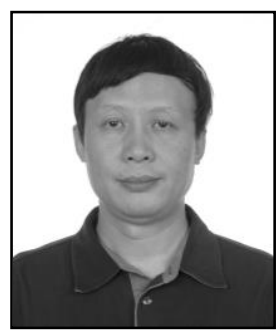

Feng Liu, is currently a Professor and also the Director of Network Management Research Center in the School of Computer and Information Technology at Beijing Jiaotong University, China. His research interests include Worldwide Interoperability for Microwave Access, Wireless Sensor Networks, network QoS, and network management systems. Email: Feng Liu @bjtu.edu.cn 